\title{
2D-SSA BASED MULTISCALE FEATURE FUSION FOR FEATURE EXTRACTION AND DATA CLASSIFICATION IN HYPERSPECTRAL IMAGERY
}

\author{
Hang Fu ${ }^{12}$, Genyun Sun ${ }^{12 *}$, Jinchang Ren ${ }^{3}$, Jamie Zabalza ${ }^{3}$, Aizhu Zhang ${ }^{12}$, Yanjuan Yao \\ ${ }^{1}$ College of Oceanography and Space Informatics, China University of Petroleum (East China), Qingdao, Shandong, \\ 266580, China \\ ${ }^{2}$ Laboratory for Marine Mineral Resources, Qingdao National Laboratory for Marine Science and Technology, \\ Qingdao, 266237, China \\ ${ }^{3}$ Department of Electronic and Electrical Engineering, University of Strathclyde, Glasgow, G11XW, United Kingdom \\ ${ }^{4}$ Satellite Environment Center, Ministry of Environmental protection of China, Beijing, 100094, China.
}

\begin{abstract}
Singular spectrum analysis (SSA) and its 2-D variation (2DSSA) have been successfully applied for effective feature extraction in hyperspectral imaging (HSI). However, they both cannot effectively use the spectral-spatial information, leading to a limited accuracy in classification. To tackle this problem, a novel 2D-SSA based multiscale feature fusion method, combining with segmented principal component analysis (SPCA), is proposed in this paper. The SPCA method is used for dimension reduction and spectral feature extraction, while multiscale 2D-SSA can extract abundant spatial features at different scales. In addition, a postprocessing via SPCA is applied on fused features to enhance the spectral discriminability. Experiments on two widely used datasets show that the proposed method outperforms two conventional SSA methods and other spectral-spatial classification methods in terms of the classification accuracy and computational cost.
\end{abstract}

Index Terms-segmented principal component analysis (SPCA), hyperspectral imagery (HSI), Multiscale 2D-SSA, feature extraction, data classification.

\section{INTRODUCTION}

Hyperspectral imagery (HSI) provides detailed spectral and spatial information simultaneously [1]. With numerous contiguous spectral bands, HSI can potentially identify different objects by detecting minor changes, which enables HSI being successfully applied for many areas such as landcover analysis in remote sensing. However, the high dimensions of HSI lead to a dimensional curse, and the data redundancy and noise are also unavoidable, which bring obstacles to the classification. Therefore, effective spectralspatial feature extraction is essential for land cover analysis.

Recently, a time-series analysis technique, singular spectrum analysis (SSA), has been introduced for feature extraction in HSI and demonstrated a good ability and effectiveness, being able to remove high-frequency noise in HSI pixels, leading to a smoothing effect [2]. Since SSA implements in the spectral domain, its improvement on classification performance is confined. While its 2-D version (2D-SSA), which is implemented in the spatial domain, can effectively utilize spatial information and explore global spatial correlation of HSI, achieving a great accuracy improvement in classification [3]. However, it cannot sufficiently use the abundant spectral content. In addition, for a given band image, its embedding window is usually fixed, which may lead to the under-smoothed or over-smoothed results of land cover.

In recent years, spectral-spatial feature fusion methods are preferred for HSI classification. Some image fusion frameworks, such as edge-preserving filtering-based (EPF) [4] multiscale feature extraction and intrinsic image decomposition (IID) [5] method, utilize and fuse abundant spatial information to improve the classification accuracy effectively. Besides, considering the deeper levels of features for classification improvement, some deep learningbased spatial-spectral methods are proposed, including feature-learning convolutional neural network (FL-CNN) [6] and GF-FSAE network [7], in which spatial-spectral fused features are learned for HSI classification.

Inspired by the above methods, a novel feature fusion method with the SPCA and 2D-SSA framework is proposed for HSI classification in this paper, which can utilize abundant spectral and multiscale spatial features. Experiments on two datasets show that the proposed method achieves better classification performance than comparison methods and provides satisfactory results.

\section{METHODOLOGY}

The flowchart of the proposed method is presented in Fig.1, which can be summarized into three steps: SPCA-based dimension reduction, multiscale 2D-SSA, feature fusion and SVM-based classification. The details are given as below.

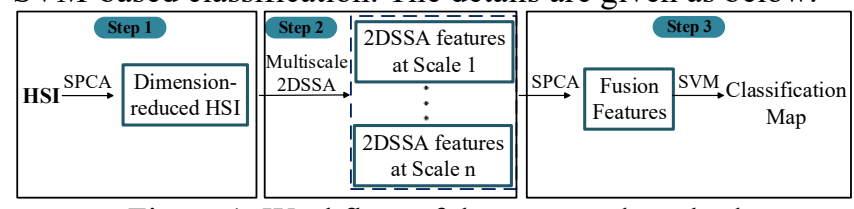

Figure 1. Workflow of the proposed method

2.1. SPCA-based dimension reduction

This is a peer-reviewed, accepted author manuscript of the following article: Fu, H., Sun, G., Ren, J., Zabalza, J., Zhang, A., \& Yao, Y. (2020). 2D-SSA based multiscale feature fusion for feature extraction and data classification in hyperspectral imagery. In IGARSS 2020 - 2020 IEEE International Geoscience and Remote Sensing Symposium (pp. 76-79). (International Geoscience and Remote Sensing Symposium (IGARSS)). IEEE. https://doi.org/10.1109/IGARSS39084.2020.9323776 
PCA can preserve most spectral features of the HSI in a few principal components by a linear transformation, and this transformation is given as follows:

$$
H_{P C A}=P C A(H)
$$

where $H$ is the hyperspectral imagery which consists of $N$ pixels and $B$ spectral bands, or a $B$-dimensional vector for each pixel, and $H_{P C A}$ is the feature data after dimension reduction, which only have several principal components.

PCA can effectively achieve the dimension reduction but it is quite sensitive to spectral noise. Therefore, the segmented PCA [8] is employed to reduce the effect of noise and extracts local spectral information. Firstly, the spectral bands of a hyperspectral imagery $H$ are divided into $K$ subsets, each of which consists adjacent bands. The number of bands $B_{k}$ for each subset $S_{k}$ is as follow:

$$
\left\{\begin{array}{c}
B_{k}=[B / K] \\
B_{K}=B-B_{k} \times(K-1)
\end{array} \quad k \in\{1,2, \ldots, K-1\}\right.
$$

where $B_{k}$ represents the value of the largest integer that is not greater than $B / K$. Then, PCA is employed to each adjacent subset as follows:

$$
H_{k}=P C A\left(S_{k}\right) \quad k=\{1,2, . ., K\}
$$

Finally, the first principal components of each band subsets are stacked together, whereas the dimension reduced HSI $H_{S P C A}$ is obtained by:

$$
H_{S P C A}=\left\{H_{1}, H_{2}, \ldots, H_{K}\right\}
$$

The band subsets contain adjacent spectral bands, which have strong correlations and contain redundant information. Compared to PCA, SPCA concentrates several adjacent spectral content, not only retains the important spectral information but also eliminates spectral redundancy.

\subsection{Multiscale 2D-SSA}

In this section, we employ 2D-SSA with different embedding window scales to extract spatial information of each band image after SPCA. This method is implemented on a grayscale image as detailed below.

Given a grayscale image I with a size $\mathrm{h} \times \mathrm{w}$, define the embedding window of size $u \times v$ (with $1 \leq u \leq h, 1 \leq v \leq w$ and $u=v$ for convenience), which moves from the top left to the bottom right of the image to construct the trajectory matrix $\mathbf{X}$. The pixels in the window are expanded and joined as a vector $V \in R^{u v \times 1}$ that as a column in the trajectory matrix, which is shown below:

$$
\mathrm{X}=\left(V_{1,1}, V_{1,2}, \cdots V_{1, w-v+1}, V_{2,1}, \cdots V_{h-u+1, w-v+1}\right) \in \mathrm{R}^{u v \times(h-u+1)(w-v+1)}
$$

Note that the trajectory matrix $\mathbf{X}$ has a structure called $\mathrm{HbH}$, i.e. Hankel by Hankel. Then, the Eigenvalues of $\mathrm{XX}^{\mathrm{T}}$ and their corresponding Eigenvectors are denoted as $\left(\lambda_{1} \geq \lambda_{2} \geq \ldots \geq \lambda_{L}\right)$ and $\left(U_{1}, U_{2}, \ldots, U_{L}\right)$, respectively. The trajectory matrix can be written as follows:

$$
\begin{gathered}
\mathrm{X}=\mathrm{X}_{1}+\mathrm{X}_{2}+\cdots+\mathrm{X}_{L} \\
\left(\mathrm{X}_{i}=\sqrt{\lambda_{i}} \mathrm{U}_{i} \mathrm{~V}_{i}^{T}, \mathrm{~V}_{i}=\mathrm{X}^{T} \mathrm{U}_{i} / \sqrt{\lambda_{i}}\right)
\end{gathered}
$$

where $\mathrm{U}_{i}$ and $\mathrm{V}_{i}$ are the empirical orthogonal functions and the principal components of the trajectory matrix, respectively. Afterwards, we select $\mathrm{X}_{1}$ as an approximation to $\mathbf{X}$, mainly because it contains the most important spatial information [3]. Finally, the matrix $X_{1}$ is converted to a new image of size $h \times w$ again, as the reconstructed image, by a two-step diagonal averaging process in the matrix antidiagonals in both each block and between blocks. Note that the scale of 2D-SSA is determined by embedding window size.

Let $\mathrm{F}_{l}$ be the output feature data at the $l$ th scale of the 2D-SSA, which is calculated by:

$$
\begin{gathered}
\mathrm{F}_{l}=2 \operatorname{DSSA}\left(H_{S P C A}, u_{l} \times v_{l}\right) \quad l=1, \ldots, n \\
\mathrm{~F}=\left\{\mathrm{F}_{1}, \ldots, \mathrm{F}_{n}\right\}
\end{gathered}
$$

where $n$ is the number of total scales for different embedding windows, and the size of $F_{l}$ is exactly the same as $H_{S P C A}$. The obtained features can capture the multiscale spatial information of the hyperspectral image effectively, where these spatial features of different scales can supplement the feature information of different land covers to achieve better recognition performance.

\subsection{Feature fusion and classification}

After the multiscale 2D-SSA, the dimension of stacked features from different scales will have a inevitable increase of the computational cost. Redundant information might exist in the staked data. In addition, the resulted images obtained by different 2D-SSA scales are continuous smooth and some texture information is removed, which have a negative effect on the classification performance because the differences of pixels belonging to different classes are reduced distinctly.

Therefore, SPCA is applied for featured images at each scale again to solve the problem presented above, which is calculated by:

$$
\begin{gathered}
Z_{l}=P C A\left(\mathrm{~F}_{l}, p\right) \quad l=\{1, \ldots, n\} \\
Z=\left\{Z_{1}, \ldots, Z_{n}\right\}
\end{gathered}
$$

where $p(p<K)$ represents the number of dimension after SPCA. Then, different scaled images after SPCA are stacked together according to (9) and used as the input of the SVM classifier. Based on margin criterion, SVM shows high robustness to dimensional problems such as the Hughes phenomenon. Moreover, SVM supports different types of kernel functions, following the suggestions from others, the RBF kernel is used in our SVM classifier [9].

\section{EXPERIMENTS AND RESULTS}

\subsection{Datasets and Experimental setup}

Two well-known HSI datasets with available ground truth, Indian Pines and Pavia University, are used in our experiments. The Indian Pines dataset has a spatial size of $145 \times 145$ pixels and contains 220 contiguous spectral bands (normally reduced to 200) and including 16 land-cover classes in the ground truth. The second dataset, Pavia University, has a spatial size of $610 \times 340$ pixels and 115 spectral bands (reduced to 103), with 9 land-cover classes. 
In addition, the SVM classifier is implemented by the LIBSVM library [10] using a RBF kernel with a five-fold cross validation, and $4 \%$ and $0.4 \%$ randomly selected samples per class for two datasets are taken as training set, being the rest of samples used for testing, respectively. The overall accuracy (OA), average accuracy (AA), kappa coefficient and computation time (the time used for feature extraction) are utilized to evaluate the proposed method.

\subsection{Scales of $2 \mathrm{D}-\mathrm{SSA}$}

In order to analyze the effect of each 2D-SSA scale in our method, an experiment is performed on Indian Pines. According to [3], the embedding window scales for 2D-SSA are chosen within $\{5,10,20\}$. In this experiment, the raw data, the feature extraction data by single scale and multiscale 2D-SSA are combined with the SVM classifier, in which the parameter $K=10$ and $p=7$ in SPCA, and the classification results are given in Table I.

Table I Classification results using the raw data and different 2D-SSA scales

\begin{tabular}{cccccc}
\hline & Raw & Scale $\{5\}$ & Scale $\{10\}$ & Scale $\{20\}$ & Multiscale \\
\hline OA & 71.99 & 91.50 & 92.55 & 92.76 & $\mathbf{9 6 . 2 5}$ \\
AA & 60.68 & 91.01 & 93.07 & 90.28 & $\mathbf{9 6 . 8 6}$ \\
Kappa & 0.68 & 0.90 & 0.92 & 0.92 & $\mathbf{0 . 9 6}$ \\
\hline
\end{tabular}

As shown in Table I, our classification framework can improve the accuracy compared to cases of raw data and three single scale. Moreover, the combination of different 2D-SSA scales can further improve the classification accuracy. This demonstrates that spatial features fusion has a potential for improved classification of HSI.

\subsection{Comparision with other approaches}

To validate the efficacy of the proposed method, the original hyperspectral data plus SVM classifiers, as well as SSA [2], EPF [4], IID [5], FL-CNN [6], GF-FSAE [7] and our method are compared. For a fair comparison, the optimal parameters of different methods are utilized in our experiment as according to [2],[4]-[7]. The parameters of proposed method are the same as section 3.2, i.e. $K=10$, $p=7$ and three scales $\{5,10,20\}$ in multiscale 2D-SSA. Moreover, the related parameters $\gamma$ and $\mathrm{C}$ of the RBF kernel are fixed at 0.125 and 1000 , respectively. The classification results and maps of the comparison approaches are shown in Tables II-III and Figs. 2-3.

As seen in Tables II-III, the classification accuracy of SSA is limited due to using only the spectral information. The EPF and IID achieve higher classification accuracy mainly because they utilize spatial features. For the other two deep learning-based methods, although they simultaneously extract spectral and spatial features, their classification accuracy improvements are limited mainly due to the limited training samples. Our feature fusion method has helped to achieve high classification accuracy in almost all classes for the Indian Pines dataset, which validates the merit of our proposed method. For Pavia University, our method also performs well in most of classes compared with other methods, while the AA is lower than IID method.

Table II Classification results of Indian Pines

\begin{tabular}{cccccccc}
\hline No. & SVM & SSA & EPF & IID & $\begin{array}{c}\text { FL- } \\
\text { CNN }\end{array}$ & $\begin{array}{c}\text { FSAE } \\
\text { FSA }\end{array}$ & Our \\
\hline 1 & 0.00 & 86.36 & 87.65 & 90.22 & 88.41 & $\mathbf{9 7 . 5 7}$ & 95.45 \\
2 & 74.23 & 82.48 & 92.17 & 92.57 & 89.82 & 85.68 & $\mathbf{9 4 . 3 8}$ \\
3 & 51.26 & 72.86 & 90.48 & 92.61 & 83.98 & 90.50 & $\mathbf{9 7 . 3 6}$ \\
4 & 22.47 & 41.41 & 58.26 & 82.96 & 87.93 & 68.22 & $\mathbf{9 4 . 7 1}$ \\
5 & 69.55 & 86.61 & 97.79 & 95.82 & 94.28 & 78.98 & $\mathbf{9 8 . 2 7}$ \\
6 & 89.43 & 88.29 & 96.37 & 98.42 & 97.25 & 95.11 & $\mathbf{9 8 . 7 1}$ \\
7 & 65.38 & 88.46 & $\mathbf{1 0 0}$ & 68.84 & 76.06 & 60.00 & $\mathbf{1 0 0}$ \\
8 & 91.48 & 97.82 & 99.47 & $\mathbf{1 0 0}$ & 99.56 & $\mathbf{1 0 0}$ & $\mathbf{1 0 0}$ \\
9 & 21.05 & 36.84 & 88.98 & 89.56 & 68.87 & 44.44 & $\mathbf{1 0 0}$ \\
10 & 66.24 & 73.63 & 70.71 & 88.77 & 87.35 & 83.43 & $\mathbf{8 9 . 1 7}$ \\
11 & 78.82 & 75.98 & 89.28 & 96.37 & 96.42 & 95.24 & $\mathbf{9 7 . 8 4}$ \\
12 & 44.11 & 60.28 & 81.07 & 89.04 & 90.58 & $\mathbf{9 1 . 1 7}$ & 86.12 \\
13 & 92.35 & 93.37 & $\mathbf{9 9 . 7 3}$ & 99.35 & 91.06 & 91.30 & 98.47 \\
14 & 90.12 & 91.02 & 98.28 & 99.32 & 99.17 & 97.01 & $\mathbf{9 9 . 5 9}$ \\
15 & 39.19 & 37.84 & 79.70 & 94.93 & 89.51 & 95.98 & $\mathbf{9 9 . 7 3}$ \\
16 & 75.28 & 77.53 & 81.14 & 98.73 & 93.59 & 86.75 & $\mathbf{1 0 0}$ \\
\hline OA & 71.99 & 77.88 & 87.37 & 94.31 & 92.35 & 85.09 & $\mathbf{9 6 . 2 5}$ \\
AA & 60.68 & 74.42 & 88.19 & 92.32 & 89.62 & 90.89 & $\mathbf{9 6 . 8 6}$ \\
Kappa & 0.68 & 0.75 & 0.86 & 0.94 & 91.07 & 0.90 & $\mathbf{0 . 9 6}$ \\
\hline & & & & & & & \\
\hline
\end{tabular}

Table III Classification results of Pavia University

\begin{tabular}{cccccccc}
\hline No. & SVM & SSA & EPF & IID & $\begin{array}{c}\text { FL- } \\
\text { CNN }\end{array}$ & $\begin{array}{c}\text { GF- } \\
\text { FSAE }\end{array}$ & Our \\
\hline 1 & 80.75 & 79.21 & 96.10 & 91.40 & 96.77 & 81.90 & $\mathbf{9 7 . 4 0}$ \\
2 & 93.38 & 91.77 & 94.60 & 99.19 & 97.48 & 97.72 & $\mathbf{9 9 . 7 4}$ \\
3 & 75.60 & 77.37 & 84.08 & $\mathbf{9 4 . 1 1}$ & 77.03 & 75.96 & 81.82 \\
4 & 77.91 & 71.91 & 71.58 & 88.84 & 87.63 & 81.61 & $\mathbf{9 0 . 4 6}$ \\
5 & 99.10 & 98.95 & 95.29 & 99.24 & 97.79 & 99.49 & $\mathbf{1 0 0}$ \\
6 & 66.35 & 56.67 & 62.83 & 98.40 & 78.68 & 86.36 & $\mathbf{9 5 . 2 5}$ \\
7 & 68.81 & 62.46 & 78.88 & $\mathbf{9 7 . 2 8}$ & 66.69 & 78.90 & 90.41 \\
8 & 76.30 & 76.14 & 85.31 & 82.64 & 85.92 & 70.89 & $\mathbf{9 6 . 1 0}$ \\
9 & 99.68 & 99.68 & 96.90 & 92.02 & $\mathbf{9 9 . 8 9}$ & 62.03 & 69.99 \\
\hline OA & 84.35 & 81.71 & 84.05 & 94.67 & 90.79 & 86.60 & $\mathbf{9 6 . 0 5}$ \\
AA & 81.99 & 79.35 & 85.06 & $\mathbf{9 3 . 6 8}$ & 87.54 & 81.65 & 91.24 \\
Kappa & 0.79 & 0.76 & 0.80 & 0.93 & 0.88 & 0.83 & $\mathbf{0 . 9 5}$ \\
\hline
\end{tabular}

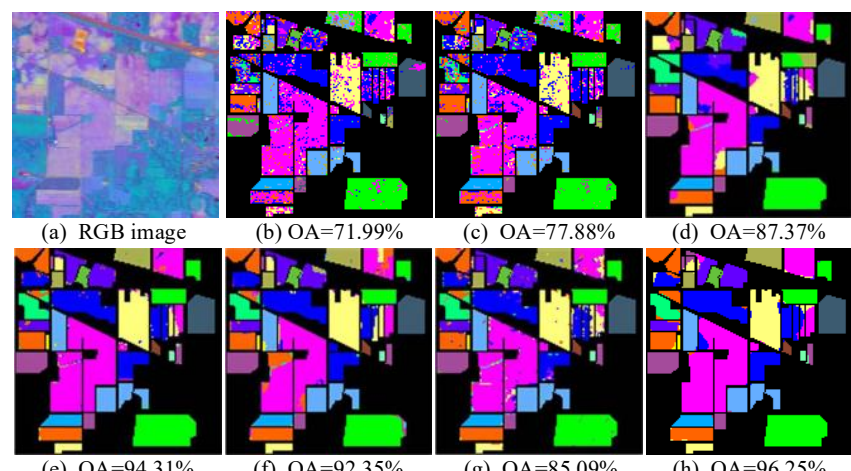

(e) $\mathrm{OA}=94.31 \%$ (g) $\mathrm{OA}=85.09 \%$ (h) $\mathrm{OA}=96.25 \%$ 
Figure. 2. Indian Pines. (a) RGB image; Results obtained by: (b) SVM; (c) SSA; (d) EPF; (e) IID; (f) FL-CNN; (g) GF-FSAE; (h) Our proposed method.

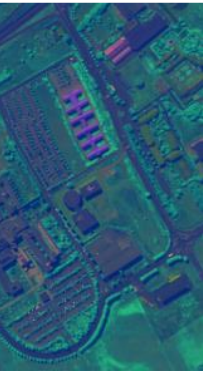

(a) RGB image

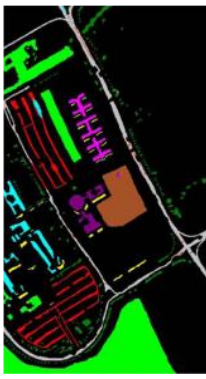

(e) $\mathrm{OA}=94.67 \%$

Figure. 3. Pavia Uni
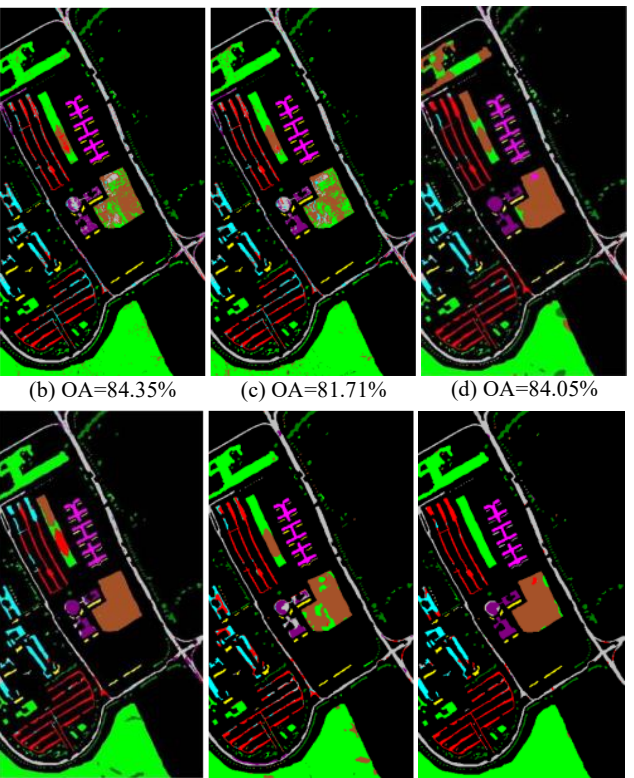

(c) $\mathrm{OA}=81.71 \%$ (d) $\mathrm{OA}=84.05 \%$

SSA; (d) EPF; (e) IID; (f) FL-

CNN; (g) GF-FSAE; (h) Our proposed method.

Figs. 2 and 3 show a visual map of the classified pixels in two datasets by using different approaches. The raw SVM and SSA have many misclassified pixels within different land covers. EPF has reduced internal misclassification of land covers, yet there still exist misclassified objects between land covers. The IID method cannot distinguish well between meadows and trees. FL-CNN and GF-FSAE cannot classify the strip features well. Our proposed method, thanks to the fused multiscale spatial information, it can achieve the best performance.

Table IV Computation time of different methods on the Indian Pines dataset $(145 \times 145 \times 200)$

\begin{tabular}{cccccccc}
\hline & SSA & 2DSSA & EPF & IID & FL-CNN & GF-FSAE & Proposed \\
\hline Time(s) & 51 & 46 & 101 & 48 & 480 & 248 & $\mathbf{1 2}$ \\
\hline
\end{tabular}

In addition, the computation time of different methods by using MATLAB on a Computer with 3.5-GHz CPU and 8-GB memory are also given in Table IV, taking Indian Pines as an example. As can be seen, our method is indeed computationally efficient, mainly because SPCA can effectively reduce the dimension and improve the computation efficiency.

\section{CONCLUSIONS}

In order to effectively utilize the spectral-spatial information, in this paper, a novel SPCA and 2D-SSA based multiscale feature fusion method is proposed for the hyperspectral image classification. The SPCA method can reduce the dimension and retain primary spectral feature of HSI. In spatial domain, the multiscale 2D-SSA is used for feature extraction, which can effectively balance the lack of extracted spatial features in a single scale. By applying SPCA again to the fused features, the spectral discriminability of pixels can be further enhanced. Experimental results on twodatasets have validated the efficacy of the proposed method, which outperforms the comparison methods and results the best classification performance with a less computational cost.

\section{ACKNOWLEDGMENT}

This work was supported by the National Natural Science Foundation of China (41971292, 41871270 , 41801275).

\section{REFERENCES}

[1] X. Jia, B. Kuo, and M. M. Crawford, "Feature Mining for Hyperspectral Image Classification," Proceedings of the IEEE, vol. 101, no. 3, pp. 676-697, 2013.

[2] J. Zabalza, R. Jinchang, W. Zheng, S. Marshall, and W. Jun, "Singular Spectrum Analysis for Effective Feature Extraction in Hyperspectral Imaging," IEEE Geoscience and Remote Sensing Letters, vol. 11, no. 11, pp. 1886-1890, 2014.

[3] J. Zabalza et al., "Novel Two-Dimensional Singular Spectrum Analysis for Effective Feature Extraction and Data Classification in Hyperspectral Imaging," IEEE Transactions on Geoscience and Remote Sensing, vol. 53, no. 8, pp. 4418-4433, 2015.

[4] X. Kang, X. Xiang, S. Li, and J. A. Benediktsson, "PCA-Based Edge-Preserving Features for Hyperspectral Image Classification," IEEE Transactions on Geoscience and Remote Sensing, vol. 55, no. 12, pp. 7140-7151, 2017.

[5] X. Kang, S. Li, L. Fang, and J. A. Benediktsson, "Intrinsic Image Decomposition for Feature Extraction of Hyperspectral Images," IEEE Transactions on Geoscience and Remote Sensing, vol. 53, no. 4, pp. 2241-2253, 2015.

[6] S. Mei, J. Ji, J. Hou, X. Li, and Q. Du, "Learning SensorSpecific Spatial-Spectral Features of Hyperspectral Images via Convolutional Neural Networks," IEEE Transactions on Geoscience and Remote Sensing, vol. 55, no. 8, pp. 4520-4533, 2017.

[7] L. Wang, J. Zhang, P. Liu, K.-K. R. Choo, and F. Huang, "Spectral-spatial multi-feature-based deep learning for hyperspectral remote sensing image classification," Soft Computing, 2016.

[8] X. Jia and J. Richards, "Segmented principal components transformation for efficient hyperspectral remote-sensing image display and classification," Geoscience and Remote Sensing, IEEE Transactions on, vol. 37, pp. 538-542, 02/01 1999.

[9] R. Archibald and G. Fann, "Feature Selection and Classification of Hyperspectral Images With Support Vector Machines," IEEE Geoscience and Remote Sensing Letters, vol. 4, no. 4, pp. 674-677, 2007. 
[10] C. Chih-Chung and L. Chih-Jen, "Libsvm: a library for support vector machines," ACM Trans. Intell. Syst. Technol., vol. 2(3), pp. 1-27, 2011. 